\title{
Simulation of Underwater Propulsion Submersible Vehicle Based on Pitch-Depth Controlling
}

\author{
$\mathrm{SHI} \mathrm{YaO}^{1, a}$, DONG Zhifeng ${ }^{2, b}$ \\ ${ }^{1,2}$ China University of Mining and Technology (Beijing), Beijing, 100083 \\ a1018063436@qq.com, bdzf@cumtb.edu.cn
}

Keywords: Submersible, Pitch-depth control, Simulation, State-space, PSO algorithm.

\begin{abstract}
The mathematical model has been built based on the designation of submersible's structure. The system parameters of the underwater propulsion submersible have been sorted, calculated and stimulated. The PSO algorithm is used to find the optimal parameters of PID controller to finish feedback control of this model. The pitch-depth double loop feedback control system has been designed to control the movement of underwater vehicle. The simulation result of the submersible control system shows that the model has high accuracy, high controllability and high control stability.
\end{abstract}

\section{Introduction}

The development of autonomous underwater vehicles (AUVs) is very rapid [1]. Much of the research and development efforts to date have been found both on defense objectives and commercial projects [2].There are two kind of underwater vehicles basically. One type is to be controlled by changing the rudder angle with simple and effective mode of control, but suffering the low accuracy of controlling. The other one is dominated by vector propulsion, and it can achieve the micro adjustment of submersible attitude. This paper designed an underwater vehicle that could move effectively and achieve micro-adjustment of attitude. If an underwater vehicle is to be completely autonomous, it must have the ability to plan paths around obstacles in order to operate safely. Many solutions to the problem of planning the path of a robot around obstacles have been proposed [3][4][5]. The designed shape of underwater vehicle is face symmetry shown in Fig.1. The mass of underwater vehicle is no more than $12 \mathrm{~kg}$, and the length of body is $0.8 \mathrm{~m}$, the diameter of body is $0.2 \mathrm{~m}$.

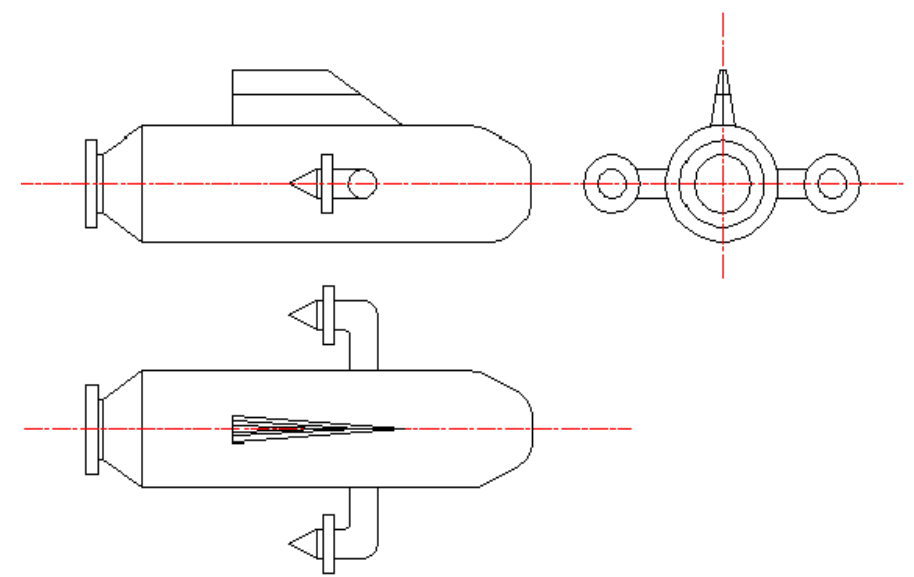

Fig.1 The shape of underwater vehicle

\section{The controlling of pitch angle}

In order to make accuracy control, a simple open-loop control module is designed to let the submersible to achieve the stable circular motion. The stimulation is as following, the right thruster is working, and the left one is not. In this way, the underwater vehicle will move in anticlockwise. It is 
obvious that stimulation results match the real condition, and the diameter of circular motion is also match the theoretical results. Therefore, this model enjoys some degree of accuracy.

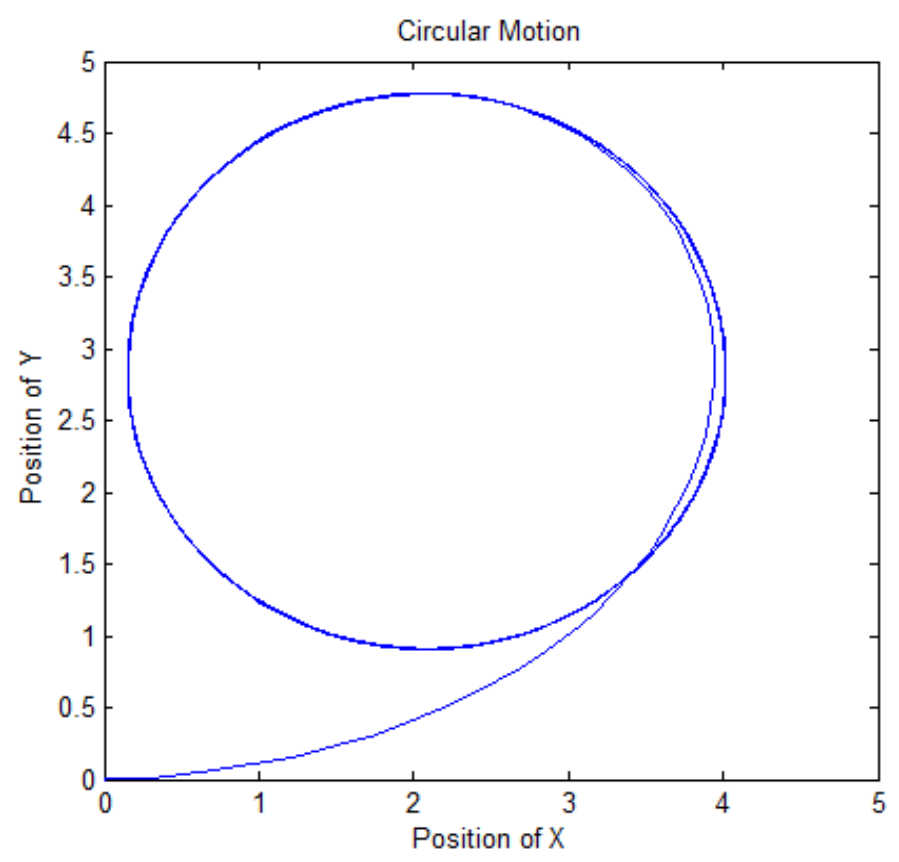

Fig.2 Circular Motion

Modelling. It is the most important to control pitch in controlling the motion of underwater vehicle. The pitch-depth control model is built. The deduction shows that the state-space is a fake time-varying system. Since the existence of matrix for coordinate transform, the state-space will change along with the change of state variable. Therefore, it is difficult to get the transfer function of system. It is decided to use PID controller, and to use the PSO algorithm to optimize the PID parameters. The general expression form of PID controller is as following,

$$
u(t)=\mathrm{K}_{p} e(t)+\mathrm{K}_{i} \int_{0}^{t} e(\tau) d \tau+\mathrm{K}_{d} \frac{d e(t)}{d t}
$$

The properties of PID controller depend on the three parameters. In the previous, it is found that if the pitch is too large, the underwater vehicle will receive the more resistance, and the submersible will be very unstable. Therefore, it is decided to control the pitch to belong the section of \pm 45 . This paper uses the property of ITAE to quality the system's property. The definition of ITAE is as following,

$$
I=\int_{0}^{\infty} t|e(t)| d t
$$

Let the PID controller of pitch to become the subsystem insert the whole mathematical model, then the closed-loop feedback controlling system has benn built. Through the PSO algorithm's optimization, the PID parameters have been determined,

$$
\mathrm{K}_{p} 45.2864, \mathrm{~K}_{i}=251.4045, \mathrm{~K}_{d}=201.4557
$$

Simulation. In the condition of pitch angle are 45 degrees and 60 degrees, the simulation results as shown in Fig.3. Under the condition of closed-loop feedback model, Fig.3 shows that the underwater vehicle achieves stable motion at 45 degrees pitch. At next, there is necessary to see the response of the pitch, and the curve is shown in Fig.4. It is shown that the changes of pitch tend to steady after a small overshoot as in the Fig. 4, and it represents that the pitch has been controlled. The analysis 
about the vector angle of the thruster has been made, and the curve is shown in Fig.5.

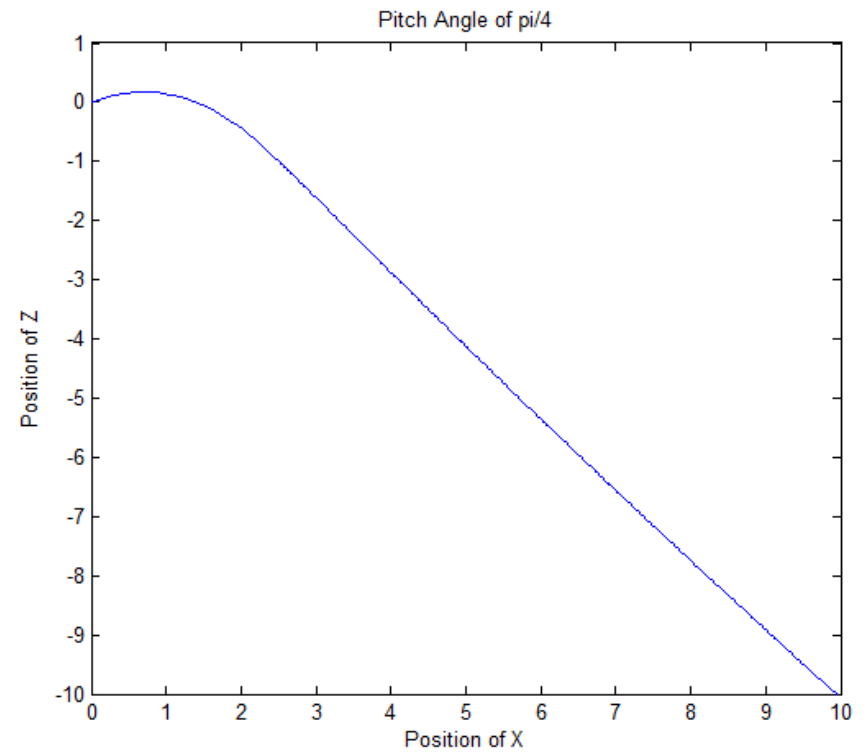

Fig.3 Pitch angle of 45 degrees

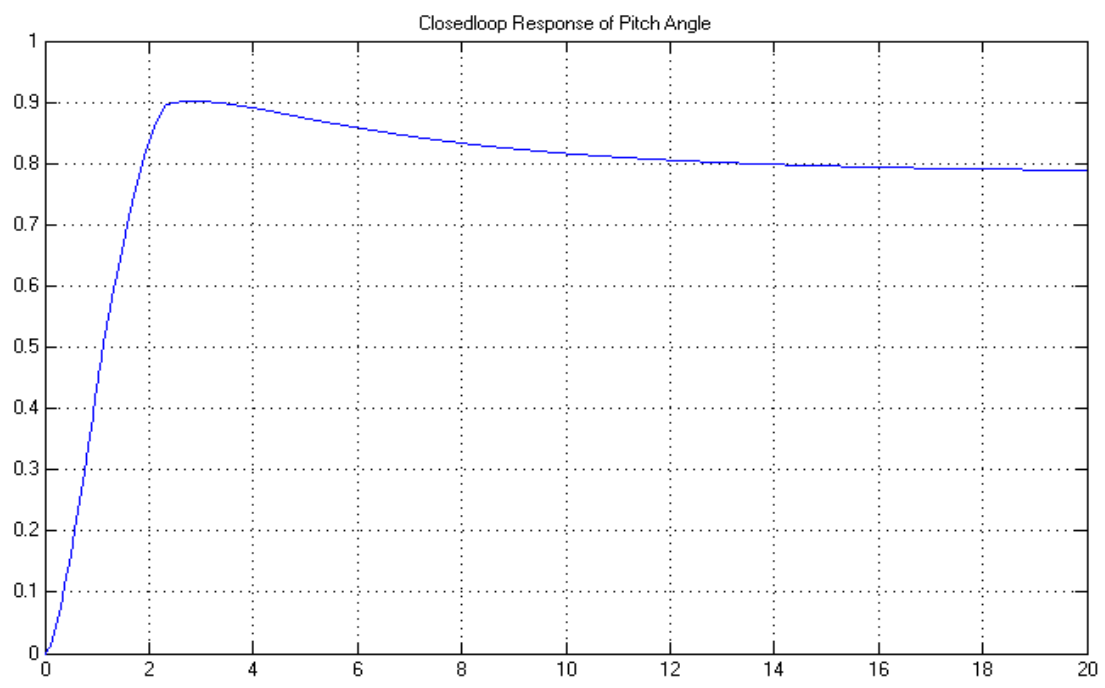

Fig.4 Closed-loop Response for Pitch angle of 45 degrees 


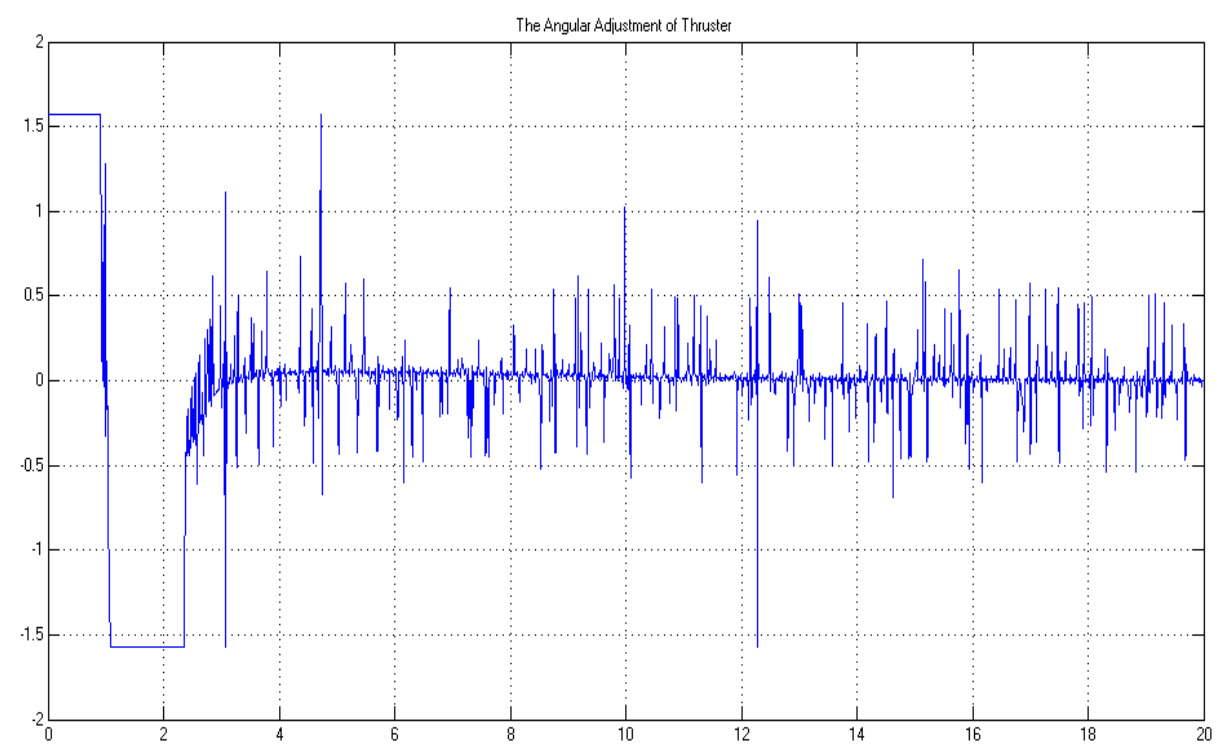

Fig.5 Angular Adjustment of Thruster

At the beginning of controlling the pitch, there is a process of fast adjustment, which generates the overshoot of the pitch. However, this process is very fast, and the pitch controlling is very steady and the time needed to steady is pretty short.

At next, the condition of controlling the pitch to 60 degrees will be concerned. In the previous, the underwater vehicle is very unstable, so the pitch value of expectation is set to 60 degrees. The simulation result has been analyzed, and the whole motion of the underwater vehicle is shown in Fig.6.

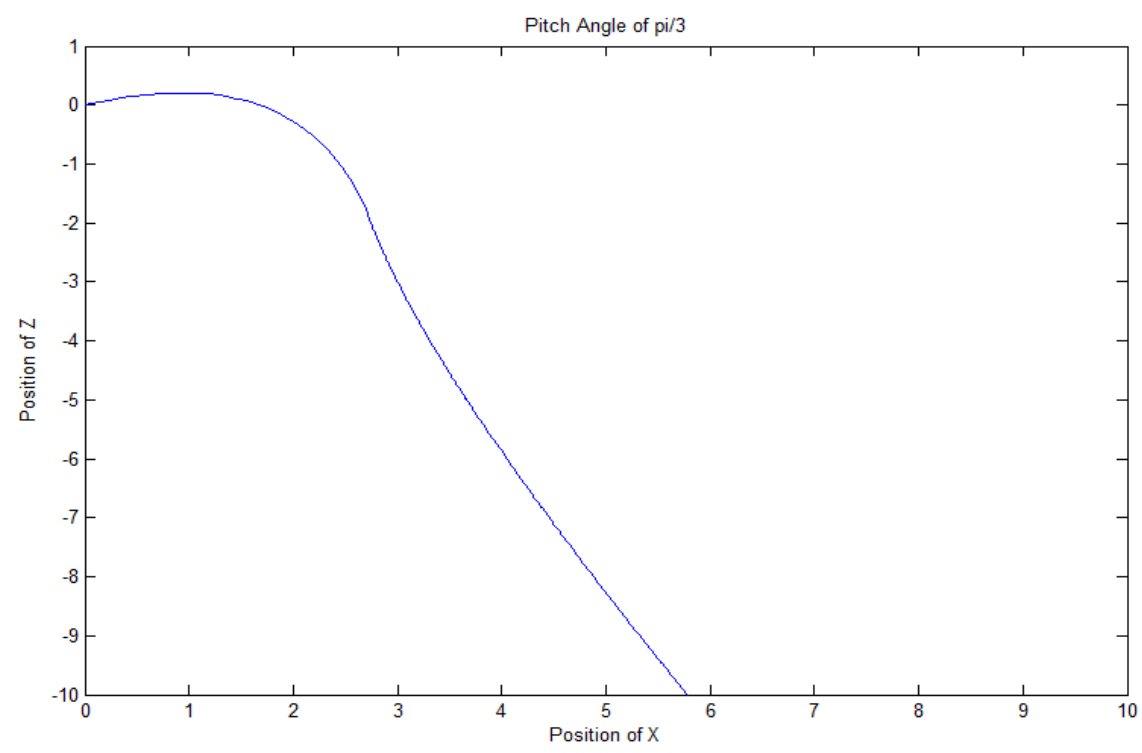

Fig.6 Pitch angle of 60 degrees

The Fig. 6 shows that although the submersible achieves the pitch control eventually, the adjustment of angle of pitch is very unsteady at the beginning. This condition will let the submersible to be controlled uneasily if there is the influence of current. In order to avoid this condition, it is decides to avoid to merge too large of pitch when designing the movement of underwater vehicle.

To continue this analysis, the curve of pitch response has been drawn in Fig.7. 


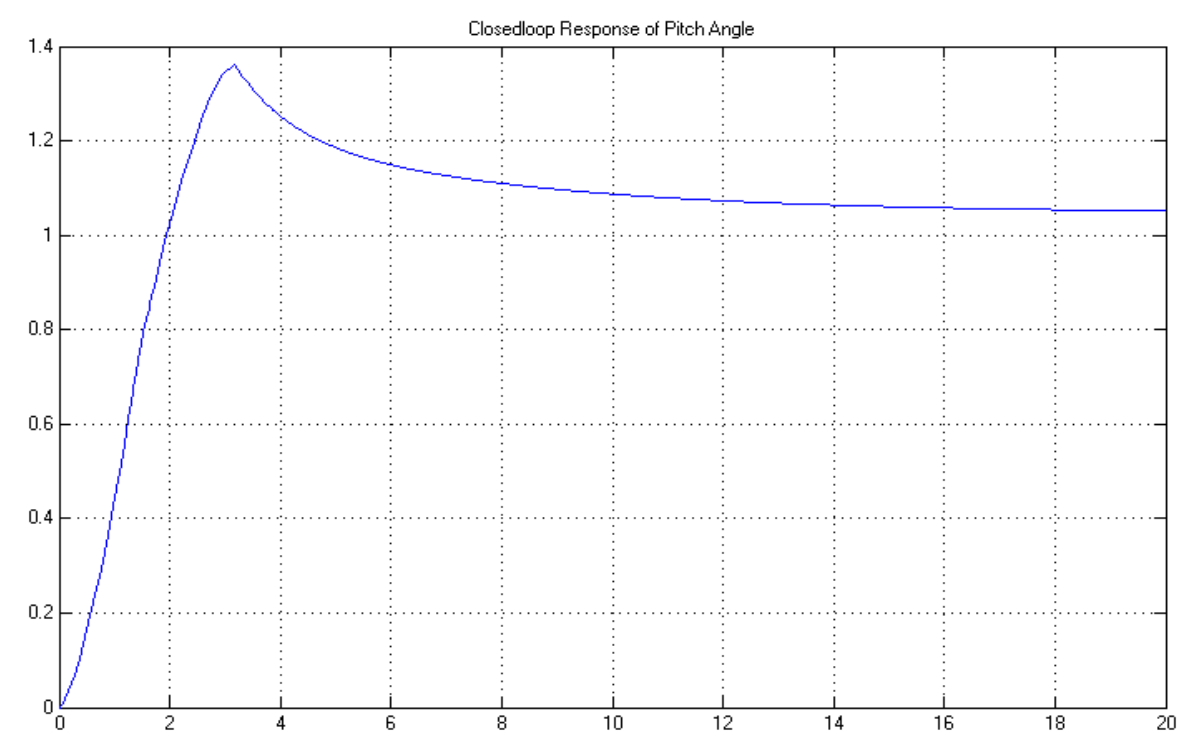

Fig.7 Closed-loop Response for Pitch Angle of 60 degrees

It is shown in Fig. 7 that the steady of this curving is 60 degrees, however, the largest pitch in this process should be concerned, which the pitch's overshoot is very seirous. The stimulation results show that the largest pitch is near 90 degrees. It is obvious that this kind of control type is catastrophic. Since it is not yet get to steady, the underwater vehicle will become out of control in the real control process. Therefore, it is necessary to limit the amplitude of the pitch in order that submersible can achieve certain pitch control and keep this pitch to move.

\section{The controlling of depth}

There is a foundation of achieving the control of depth because of single vector of the main thruster. The orientation of main thruster is along with the $\mathrm{x}$ axis of body-fixed coordinate, so the pitch of the underwater vehicle will ensure the direction of the movement. The depth feedback control model is designed based on pitch-depth double loop.

In order to control the depth, the first step is to get the current depth, then, to compare this depth value with the expectation ones, at next, the pitch of expectation at current has been calculated. According to the thruster's force, the pitch will be adjusted. It is obvious that when the depth error tends to zero, the pitch of expectation and the pitch of current are both to tend to zero. Finally, the control of depth would be finished. Using the feedback loop of depth to stimulate, and setting the 10 meters as the depth of expectation, the curve of response of depth is shown in Fig.8.

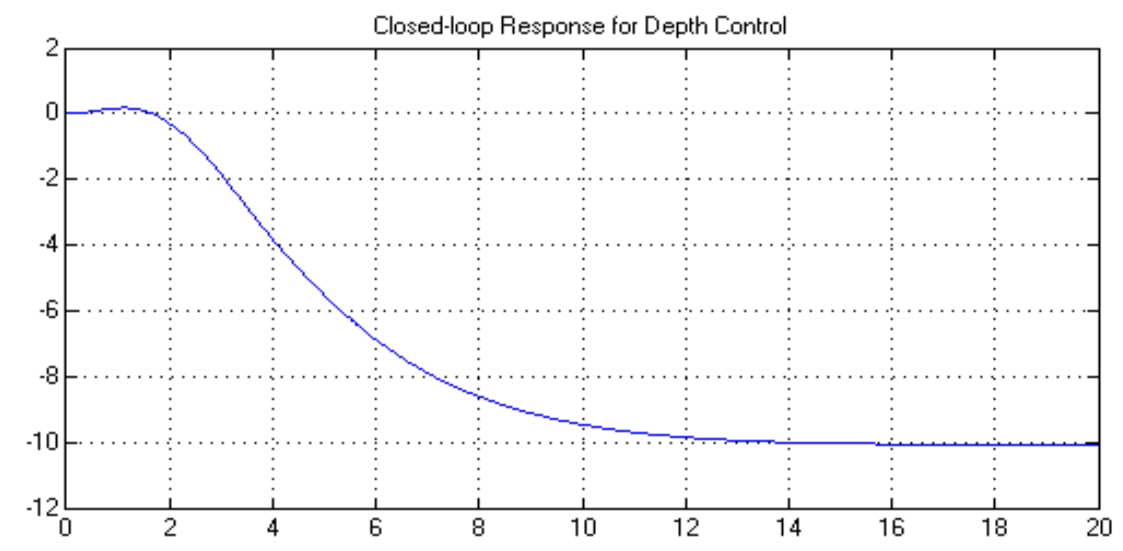

Fig.8 Closed-loop Response for Depth Control 
The Fig.8 shows that the response of depth is fast and steady, at meantime, there is no abrupt change in depth control process. It is matched the requirement of controlling. After the response of depth matches the requirement, it is necessary to consider the question that if there will be very large of pitch, and the curve is shown in Fig.9.

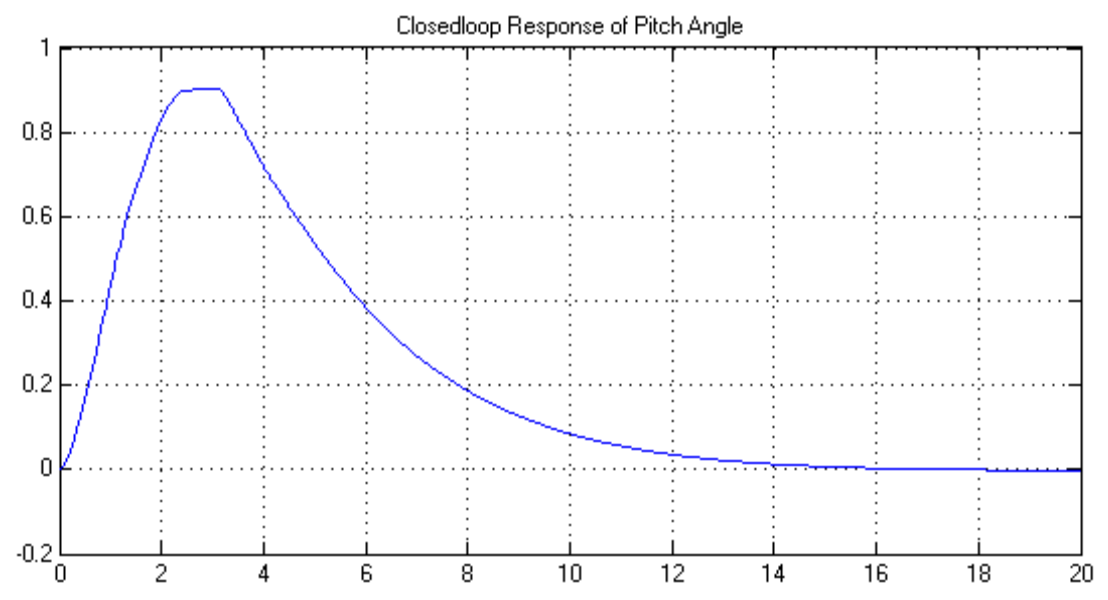

Fig.9 Pitch adjustment in depth control

From the Fig.9, the largest pitch will be 45 degrees in the control process, and the rest is very steady. It shows that the pitch will get very stable control in the process of depth control, and there will not be the risk of control. The pitch-depth double loop feedback control system can achieve stable control at the depth. The response of vector angle is shown in Fig.10.

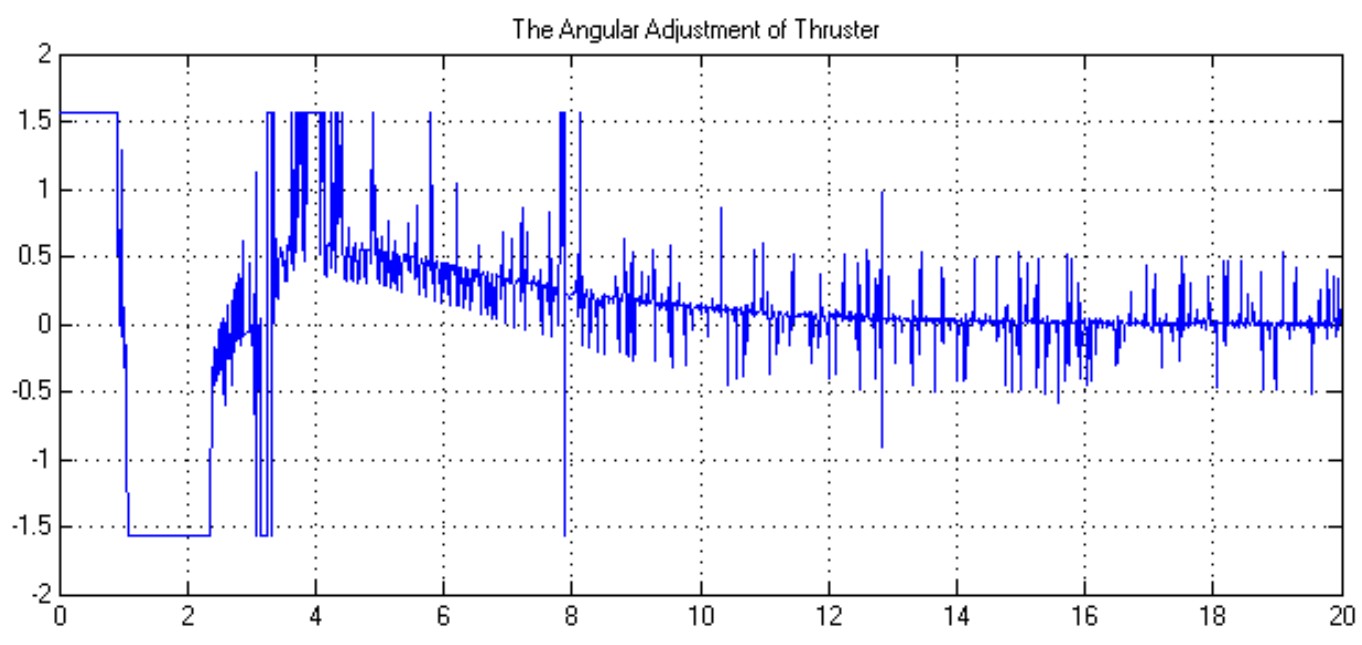

Fig.10 Thruster adjustment in Depth Control

It is shown from Fig.10 that the system needs a vector angle near 90 degrees at the beginning of control process, which this angle will generate a step response, in the consideration of the arm of force is not so long. This kind of big vector angle will only generate the response of velocity and acceleration by the gyro sensor.This is the prerequisite of control, and the controlling is very steady after this step response, which shows that the whole process is improved. The stimulation results shows that the design of control system has high accuracy.

\section{Summary}

A new model is built for submersible combined the advantage of two typical underwater vehicles. The pitch-depth double loop feedback control system has been designed to control the movement of underwater vehicle. The simulation results of the submersible control system show that the model has high accuracy, high controllability and high control stability. 


\section{Acknowledgment}

This work was financially supported by "The Fundamental Research Funds for the Central Universities” (No.2010YJ04). The authors would like to acknowledge and thank for the support.

\section{References}

[1] JJ Leonard, AA Bennett, CM Smith. Autonomous Underwater Vehicle Navigation. MIT Marine robotics Laboratory Technical Memorandum 98-1.

[2] C Kinsey, M R Eustice, L L Whitcomb. A Survey of Underwater Vehicle Navigation: Recent Advances and New Challenges (2006). Proceedings of the 7th Conference on Maneuvering and Control of Marine Craft (MCMC’2006). IFAC.

[3] J. Garus. OPTIMIZATION OF THRUST ALLOCATION IN THE PROPULSION SYSTEM OF AN UNDERWATER VEHICLE. Int. J. Appl. Math. Comput. Sci., 2004, Vol. 14, No. 4, pp461-467.

[4] Yin Liu, Tao Liu, YongHu, Design summary for the exo-structure of deep-sea manned submersible. Journal of Ship Mechanics, 2006.

[5] Inge Spangelo and Olav Egeland. Generation of energy-optimal trajectories for an autonomous underwater vehicle. Proceeding of the 1992 IEEE International Conference on Robotics and Automation Nice France May 1992 